\title{
Parallel Beam Optical Tomography
}

\author{
Liya Yu and P. C. Cheng
}

Advanced Microscopy and Image Lab., Dept of Electrical Eng., SUNY at Buffalo, New York 14260

X-ray tomography is a well established technology for visualization 3D objects. The contrast mechanism of X-ray tomography is based on the differences of X-ray absorbance of materials under study. However, soft tissues, consists of mainly light elements, produce very poor contrast under conventional short wavelength X-rays. Although there are sufficient absorption differences among $\mathrm{C}$, $\mathrm{N}, \mathrm{P}$, and $\mathrm{O}$ in the soft X-ray spectrum [1], unfortunately, the penetration thickness is also reduced to approx. $10 \mu \mathrm{m}$. In contrast, water window in the visible and near infrared (NIR) spectrum is the most transparent region [2], therefore, a tomographic system utilizes the visible and NIR spectrum could be useful for 3D imaging of living tissues. The X-ray scattering and refraction components are very small and generally neglected in tomographic reconstruction. However, when visible and NIR photons incident on a biological specimen, significant scattering and refraction could occur. Since the conventional tomographic reconstruction algorithm considers only the intensity of ballistic photons; therefore, it is necessary to construct an imaging system of which only ballistic photons are detected. Attempt on optical cone-beam tomography using visible light was reported earlier [3] by minimizing scattering and refraction effects using refractive index matched (cleared) specimen. Figure 1 is a diagrammatic representation of the optical tomographic imaging system (one path). The system consists of a basic Schlieren optical setup using two lenses/mirrors with a point light source and a pinhole detector. The pinhole acts as a spatial filter to remove those photons resulting from refraction and scattering within the specimen. Arranging multiple Schlieren optics in a circular fashion provides an imaging system capable of capturing number of projection images simultaneously (FIG 2). This reduced/eliminate the number of specimen rotation steps needed for the required large number of projection images for tomographic reconstruction. The imaging model for optical tomography is quite simple. For a two-dimensional object, the projection data are obtained as follows [4]. Let $i(x, y)$ denote the attenuation coefficient of the object at the point $(x, y)$. Assuming that the illumination is an infinitely thin parallel beam of light, the intensity of the detected light along the ray is governed by the refractive indices, scattering coefficient of the specimen and the size of detection pinhole. Then the following linear transformation is obtained:

$$
P_{\theta}(t)=\int_{L} i(x, y) d u, 0 \leq \theta \leq \pi,-\infty<t<\infty,
$$

Where $(\theta, t)$ represents the polar coordinates of the perpendicular vector from the light to the center of object (FIG 2). Theoretically, we can determine $i(x, y)$ from $P_{\theta}(t)$ exactly. In practice, only an approximate $i(x, y)$ can be estimated, because the number of available projections $P_{\theta}(t)$ is finite. The conventional way to image a three-dimensional object is to illuminate each time a specific section of an object with a narrow beam of light and use a two-dimensional reconstruction algorithm, a threedimensional reconstruction can be achieved by using a 2 dimensional CCD detector. In a polar coordinate system (FIG 3), the reconstruction algorithm as

$$
i(r, \phi)=\frac{1}{2} \int_{0}^{2 \pi} \int_{-\infty}^{\infty} P_{\theta}(t) f(r \cos (\theta-\phi)-t) d t d \theta,
$$

Where $f(t)$ is given as follows 


$$
f(t)=\int_{-\infty}^{\infty}|w| e^{j 2 \pi w t} d w,
$$

The optical tomographic system provides possibility of studying specimen consists of heterogeneous refractive indexes structures and/or scattering structures. FIGS 4-7 are projection images of Jello gel in water. Three-dimensional air-flow pattern [5], such as flame (FIG 8) or vapor from an aerosol bottle, can also be studied.

[1] P. C. Cheng et al., In: Modern microscopy. eds. P. Duke \& A. Michette. Plenum Press. (1990) 87

[2] P. C. Cheng, In: Handbook of biological confocal microscopy, $3^{\text {rd }}$ edition, ed. J. Pawley. Plenum Press (2006) 167-214

[3] P. C. Cheng, Maize Genetic Coop News Letter 69 (1995) 28

[4] G. E. Wang, Cone-beam X-ray Microtomography, PhD thesis, Dept. of Electrical and Computer Engineering, SUNY Buffalo (1992)

[5] W. Y. Cheng, et al., Microsc. Microanal., 8 (Suppl.2) (2002)1048-1049
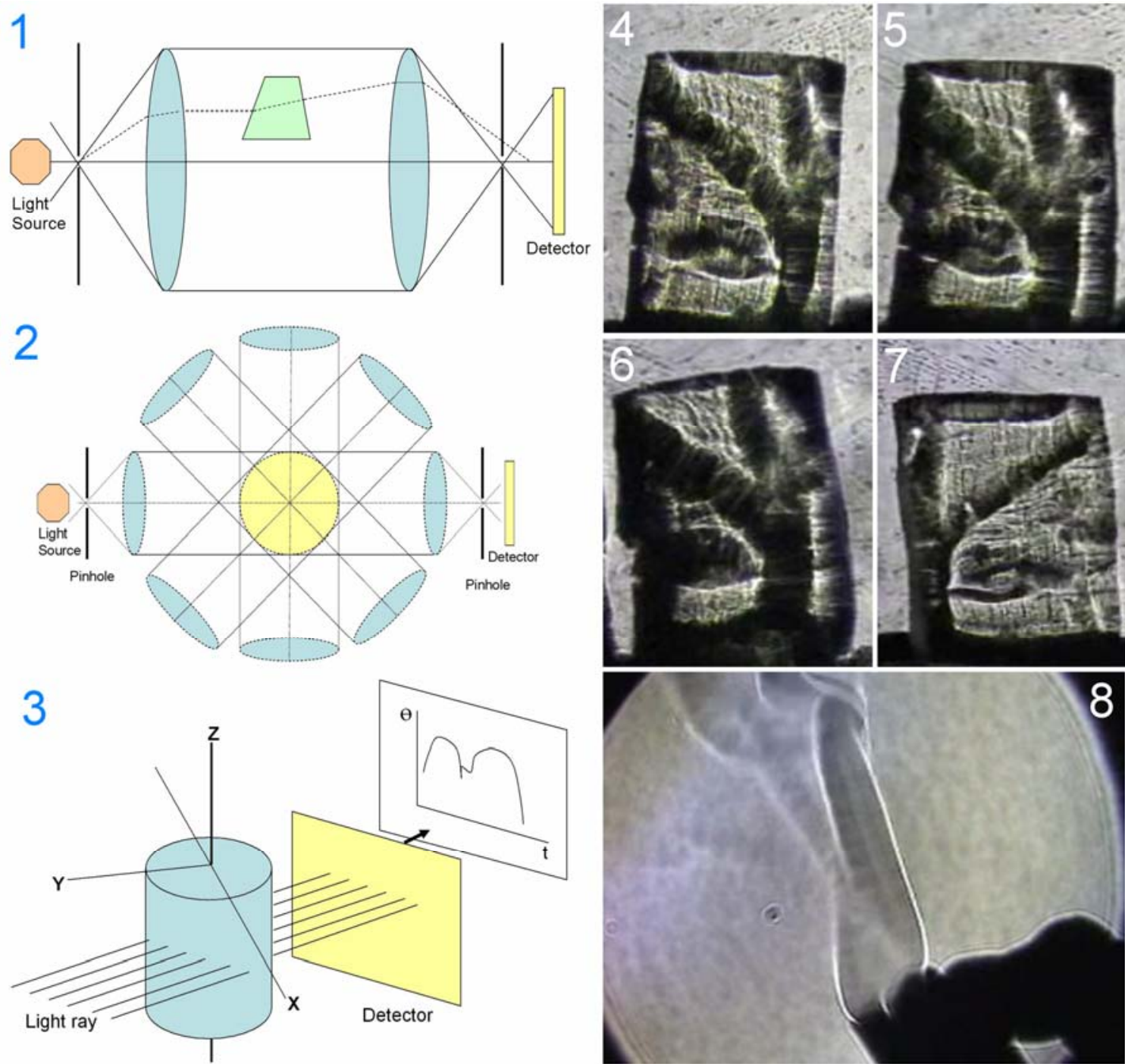

FIG 1. Schlieren optical setup for parallel-beam optical tomography. FIG 2. Four-beam tomographic system. The yellow region indicates the reconstruction volume. FIG 3. Coordinate system used in the reconstruction algorithm. FIG 4-7. Various projections of a Jello gel ; FIG 8. Flame from a lighter. 